\title{
Assessment of Lifestyle and Eating Habits among a Nationally Representative Sample of Iranian Adolescent Girls: the CASPIAN-V Study
}

Ramin Shayan-Moghadam, MD'; Motahar Heidari-Beni, PhD $^{1 *}$; Roya Riahi, MSc'; Mohammad Esmail Motlagh, MD²; Shahin Fesharaki, MD; Ramin Heshmat, MD'; Seyede Shahrbanoo Daniali, PhD'; Roya Kelishadi, MD ${ }^{1 *}$

${ }^{1}$ Child Growth and Development Research Center, Research Institute for Primordial Prevention of Non-Communicable Disease, Isfahan University of Medical Sciences, Isfahan, Iran

${ }^{2}$ Pediatrics Department, Ahvaz Jundishapur University of Medical Sciences, Ahvaz, Iran

${ }^{3}$ Student Research Committee, School of Medicine, Isfahan University of Medical Sciences, Isfahan, Iran

${ }^{4}$ Chronic Diseases Research Center, Endocrinology and Metabolism Population Sciences Institute, Tehran University of Medical Sciences, Tehran, Iran

\begin{abstract}
Background: The present study assess lifestyle and eating habits of Iranian adolescent girls.

Methods: This cross-sectional study was conducted on 3207 adolescent girls, aged 12-18 years. Lifestyle and eating habits of adolescents were assessed.

Results: The prevalence of overweight was $11.3 \%$ and that of obesity was $10.2 \%$. The frequencies of daily intake of fruits, dairy products and vegetables were $60.9 \%, 45.6 \%$ and $33.5 \%$, respectively. Skipping breakfast $(15.8 \%)$ was more frequent than skipping dinner or lunch. Moreover, $10.6 \%$ of subjects had fast food daily and $16.2 \%$ had fast food weekly. Overall, $55.2 \%$ of participants watched television for at least 2 hours a day; and $27.1 \%$ reported to have a sleeping time of less than 8 hours a day. Low physical activity, i.e. less than 30 minutes per day, was less frequent among participants with high socio-economic status (SES) compared to those with medium or low SES $(57.6 \%$ vs. $61.3 \%$ and $64.1 \%$, respectively, $P$ value $=0.010)$.

Conclusion: Improvement of lifestyle habits should be considered in public health plans for health promotion of adolescent girls and the next generation. Primordial prevention of chronic diseases by improving healthy lifestyle of adolescent girls should be a national public health priority.

Keywords: Adolescent girls, Dietary behavior, Life style, Physical activity, Prevention

Cite this article as: Shayan-Moghadam R, Heidari-Beni M, Riahi R, Motlagh ME, Fesharaki S, Heshmat R, et al. Assessment of lifestyle and eating habits among a nationally representative sample of iranian adolescent girls: the CASPIAN-V study. Arch Iran Med. 2020;23(8):522-529. doi: 10.34172/aim.2020.54.
\end{abstract}

Received: August 31, 2019, Accepted: June 2, 2020, ePublished: August 1, 2020

\section{Introduction}

Adolescence is the transitional phase of development and growth between childhood and adulthood. According to the World Health Organization (WHO) definition, any person between 10-19 years of age is considered an adolescent. ${ }^{1}$ It is a critical period of human life due to several physicals, psychological, and emotional challenges. Several studies showed that female adolescents mostly have more problems than males during this period. ${ }^{2}$ Lifestyle has a great effect on health and contributes to several nutritional, communicable, and non-communicable diseases throughout life. ${ }^{3}$ There are several changes in personal behaviors and habits during adolescence. ${ }^{4}$ Some of these alterations in adolescents' behaviors such as physical activity, dietary habits, smoking, and sleep duration have a serious effect on their health during adulthood. ${ }^{5}$ Physical activity has a significant role in mental and psychological health of adolescents. ${ }^{6}$

Sleep quality and its duration are other critical issues during adolescence which have a great effect on mental health, brain development, cognitive system and learning and memory system. Adolescents tend to sleep shorter in comparison with children. ${ }^{7}$

Adolescents tend to consume salty and fatty junk foods and most of them have unhealthy dietary habits. These foods are poor in nutrients and affect the nutritional status of females during the adolescence period and in pregnancy. Adolescents have more concerns about their body image and excess weight, especially female adolescents, which may cause failure to ingest optimal

*Corresponding Authors: Motahar Heidari-Beni, PhD; Department of Nutrition, Child Growth and Development Research Center, Research Institute for Primordił al Prevention of Non-Communicable Disease, Isfahan University of Medical Sciences, Isfahan, Iran. Fax: +983137925280, Tel. +983137925284, Email: motahar. heidari@nutr.mui.ac.ir

Roya Kelishadi, MD; Department of Pediatrics, Child Growth and Development Research Center, Research Institute for Primordial Prevention of Non-Communicable Disease, Isfahan University of Medical Sciences, Isfahan, Iran. Fax: +983137925280, Tel. +983137925281, Email: kelishadi@med.mui.ac.ir 
nutrients and energy which are necessary for rapid growth during the adolescence period. It also causes malnutrition, micronutrient deficiency and iron deficiency anemia. ${ }^{8}$

Sun exposure is another factor that is important during adolescence. It is the main source of cutaneous vitamin $\mathrm{D}$ production. Vitamin $\mathrm{D}$ is a crucial micronutrient in regulating the serum calcium level, bone metabolism, and bone health. Maintaining vitamin D in the standard level is very crucial, especially during adolescence. ${ }^{9}$ This fact shows the importance of sufficient sun exposure time for adolescents, especially adolescents girls in Iran which have less outdoor activity than boys. Adolescence is a critical period of life, especially in females. Healthy adolescent girls will become healthy adult females in the future. Adolescent girls have a significant role as future mothers in the growth and development of the next generation. So, healthy lifestyle in female adolescents is very important.

The present cross-sectional multi-center study assessed some of the main lifestyle and eating habits in Iranian adolescent girls.

\section{Materials and Methods}

Study Population

In the present study, the data of national school-based surveillance was used. The data was part of the fifth phase of the Childhood and Adolescence Surveillance and Prevention of Adult Non-communicable disease (CASPIAN-V) study. This study was performed among primary and secondary schools students ( $7-18$ years) in urban and rural areas of 30 provinces of Iran. Protocol details of the CASPIAN-V study have been explained previously. ${ }^{10}$

\section{Data Collection}

In the present study, data from 3,207 adolescent girls were assessed. The age range of participants was 12-18 years.

Demographic characteristics, weight status, physical activity, dietary habits, sleep patterns, television watching, personal computer (PC) working time, duration of mobile use and duration of sun exposure were assessed.

These data were obtained from two sets of validated questionnaires. One of the questionnaires was adopted from the World Health Organization's Global School Student Health Survey (WHO-GSHS) and was filled out through interviews with students; the second questionnaire including dietary habits, leisure time activities, and sleep pattern was completed through direct interviews with one of the parents. All questionnaires were checked for validity and reliability. ${ }^{11,12}$

Frequency of intake of dietary groups such as vegetables (fresh or cooked vegetables), fruit (fresh fruit, dried fruit, fresh fruit juice, packed fruit juice), dairy products (milk, yogurt, cheese), fast food (sausages, burgers and pizza), salty snacks (snacks, chips and pretzels), sweets (cakes, cookies, pastries, biscuits and chocolate), sugar, and drinks (carbonated drinks, diet sodas, non-alcoholic beer, tea, coffee) was asked for assessment of dietary habits.

A calibrated scale was placed on a flat ground and weight was measured to the nearest $0.1 \mathrm{~kg}$. Height was measured using a portable stadiometer to the nearest $0.1 \mathrm{~cm}$. Waist circumference was measured at the midway point between the lower border of the rib cage and the iliac crest at the end of normal expiration.

The number of hours per day watching television and/ or videos, using a personal computer or playing electronic games was asked for assessment of screen time (ST) behavior. Based on this data, the total cumulative time spent as ST was calculated. Two categories were defined for ST: less than $2 \mathrm{~h} / \mathrm{d}$ was considered as low, and $2 \mathrm{~h} / \mathrm{d}$ or more was considered as high group

The average hours per day spent for sleeping on weekdays and weekends were considered for sleep duration. Sleep duration equal to or less than $8 \mathrm{~h}$ /day was defined as short sleep duration. The onset of sleep was occasionally asked.

The data on frequency of leisure time physical activity outside the school during the preceding week was collected using a validated questionnaire. ${ }^{11}$ Students were considered having adequate physical activity if they had exercises for at least 30 minutes per day that led to sweating and a substantial increase in respiratory or heart rate. Two questions were used for assessment of physical activity. The number of days of physical activity for an overall 30 minutes per day and physical education classes at school per week were questioned. Physical activity less than 30 min per day was defined as low and physical activity more than 30 min per day was defined as sufficient. ${ }^{13}$

Evaluation of sun exposure was done using the WHO questionnaire. The parts of the body exposed to sunlight, the time of exposure to outdoor sunlight and the use of sunscreen creams on weekdays and weekends were asked

Feeling worthless, confusion, nervousness, anxiety, having a poor sleep during the 6 preceding months and feeling depressed and worried during the past 12 months were assessed by seven multiple-choice Likert-scale questions. Quantitative values of mental status score were determined by the sum of scores divided by seven. The range of mental status score was 1 to 4.71 , with higher scores showing better mental status.

The principal component method was used for socioeconomic status (SES) assessment. Jobs and education of father and mother, owning a car and computer, and type of student's school (private, public) were asked. Three categories were considered for SES: low (first tertile), medium (second tertile) and high (third tertile).

\section{Statistical Analysis}

The SPSS statistical package (SPSS, Chicago, IL; version 18:0) for windows was used for analyzing the data. Quantitative variables were shown as mean \pm standard deviation (SD). Qualitative variables were shown as 
frequency (percentage). The chi-square test was used for comparison of demographic variables and lifestyle factors among various levels of SES. $P$ values of less than 0.05 were considered as statistically significant.

\section{Results}

The demographic characteristics of the adolescent girls are shown in Table 1. The mean (SD) age of girls was 15.2 (1.72) years. The prevalence of obesity was $10.2 \%$ and abdominal obesity was $20.4 \%$ among Iranian adolescent girls.

The food and snack consumption of the adolescent girls is shown in Table 2. The frequency of daily consumption of fruits $(60.9 \%)$, vegetables $(33.5 \%)$, and dairy products $(45.6 \%)$ was higher than sweet snacks, salty snacks, carbonated drinks and fast food.

Table 3 presents the lifestyle habits of Iranian adolescent girls. Among the main meals, skipping breakfast (15.8\%) was more common than lunch and dinner. Also, 55.2\% of the girls watched television for 2 hours or more per day.

As shown in Table 4, anger (42.8\%) followed by worry $(38 \%)$ were more frequent among Iranian adolescent girl than other psychological disorders. In addition, $28.6 \%$ of the adolescent girls perceived themselves as overweight or obese while having normal weight.

The frequency of lifestyle factors of participants according to SES is shown in Table 5. Low physical activity, i.e. less than 30 minutes per day, was less frequent among adolescent girls with high SES compared to those with medium or low SES (57.6\% vs. 61.3\% and 64.1\%, respectively). The frequency of consuming food items

Table 1. Demographic Characteristics of Iranian Adolescent Girls

\begin{tabular}{|c|c|c|c|}
\hline \multicolumn{2}{|l|}{ Variables } & Mean & SD \\
\hline \multicolumn{2}{|l|}{ Age (year) } & 15.2 & 1.72 \\
\hline \multicolumn{2}{|l|}{ Weight (kg) } & 51.53 & 13.41 \\
\hline \multicolumn{2}{|c|}{ Height $(\mathrm{cm})$} & 157.10 & 9.89 \\
\hline \multicolumn{2}{|c|}{ Waist circumference $(\mathrm{cm})$} & 71.28 & 10.31 \\
\hline & & No. & $\%$ \\
\hline \multirow{2}{*}{ Living area } & Urban & 2553 & 79.6 \\
\hline & Rural & 654 & 20.4 \\
\hline \multirow{3}{*}{$\begin{array}{l}\text { Socio- } \\
\text { economic } \\
\text { status }\end{array}$} & Low & 1007 & 32.8 \\
\hline & Medium & 1002 & 32.7 \\
\hline & High & 1057 & 34.5 \\
\hline \multirow{3}{*}{$\begin{array}{l}\text { Father's } \\
\text { education }\end{array}$} & Illiterate & 388 & 12.6 \\
\hline & Diploma/under diploma & 2245 & 72.8 \\
\hline & Academic education & 449 & 14.6 \\
\hline \multirow{3}{*}{$\begin{array}{l}\text { Mother's } \\
\text { education }\end{array}$} & Illiterate & 538 & 16.9 \\
\hline & Diploma/under diploma & 2307 & 72.5 \\
\hline & Academic education & 335 & 10.5 \\
\hline \multirow{4}{*}{$\begin{array}{l}\text { Weight } \\
\text { status }\end{array}$} & Underweight & 386 & 12.1 \\
\hline & Normal weight & 2110 & 66.4 \\
\hline & Overweight & 359 & 11.3 \\
\hline & Obese & 323 & 10.2 \\
\hline \multirow{2}{*}{$\begin{array}{l}\text { Abdominal } \\
\text { obesity }\end{array}$} & No & 2553 & 79.6 \\
\hline & Yes & 654 & 20.4 \\
\hline
\end{tabular}

was not significantly different according to the SES of participants $(P$ value $>0.05)$.

\section{Discussion}

This nationwide study assessed some lifestyle habits including dietary and physical activity habits among Iranian adolescent girls.

In this study, the prevalence of overweight was $11.3 \%$ and that of obesity was $10.2 \%$. In Asian adolescents, the prevalence rates of overweight and obesity are reported at $13.7 \%$ and $6.2 \%$, respectively. ${ }^{14}$ A large study conducted among American children and adolescents showed that the prevalence of obesity was $16.9 \%$ and that of overweight was $31.8 \% .^{15}$ In a large cross-sectional survey on US youth, an escalating trend of obesity was

Table 2. Food and Snack Consumption of Iranian Adolescent Girls

\begin{tabular}{llllll}
\hline Food items & & Daily & Weekly & Rarely & Never \\
\hline \multirow{2}{*}{ Sweet snacks } & No. & 715 & 1308 & 1102 & 82 \\
& $\%$ & 22.3 & 40.8 & 34.4 & 2.5 \\
Salty snacks & No. & 205 & 877 & 1663 & 451 \\
& $\%$ & 6.41 & 27.44 & 52.03 & 14.1 \\
Carbonated drinks & No. & 100 & 770 & 1504 & 829 \\
& $\%$ & 3.1 & 24 & 47 & 25.9 \\
\multirow{2}{*}{ Fruits } & No. & 1668 & 729 & 239 & 104 \\
& $\%$ & 60.9 & 26.6 & 8.7 & 3.8 \\
Vegetables & No. & 1061 & 1480 & 439 & 186 \\
& $\%$ & 33.5 & 46.7 & 13.9 & 5.9 \\
Dairy products & No. & 1462 & 1238 & 470 & 35 \\
& $\%$ & 45.6 & 38.6 & 14.7 & 1.1 \\
Fast food & No. & 339 & 520 & 1762 & 581 \\
& $\%$ & 10.59 & 16.24 & 55.03 & 18.14 \\
\hline
\end{tabular}

Table 3. Lifestyle Habits of Iranian Adolescent Girls

\begin{tabular}{llcc}
\hline Lifestyle Habits & & No. & $\%$ \\
\hline \multirow{2}{*}{ Breakfast } & Non skipper & 2662 & 84.2 \\
& Skipper & 501 & 15.8 \\
Lunch & Non skipper & 2900 & 91.4 \\
& Skipper & 272 & 8.6 \\
Ninner & Non skipper & 2884 & 90.4 \\
& Skipper & 306 & 9.6 \\
Sleep duration & $<8 \mathrm{~h} / \mathrm{d}$ & 847 & 27.1 \\
& $\geq 8 \mathrm{~h} / \mathrm{d}$ & 2284 & 72.9 \\
Sun exposure & $<5 \mathrm{~min} / \mathrm{d}$ & 520 & 16.3 \\
& $1-30 \mathrm{~min} / \mathrm{d}$ & 1397 & 43.9 \\
& $>30 \mathrm{~min} / \mathrm{d}$ & 1267 & 39.8 \\
Television watching & $<2 \mathrm{~h} / \mathrm{d}$ & 1435 & 44.8 \\
& $\geq 2 \mathrm{~h} / \mathrm{d}$ & 1769 & 55.2 \\
\hline Personal computer use & $<2 \mathrm{~h} / \mathrm{d}$ & 2813 & 89.8 \\
& $\geq 2 \mathrm{~h} / \mathrm{d}$ & 320 & 10.2 \\
& $<2 \mathrm{~h} / \mathrm{d}$ & 2975 & 95.1 \\
\hline Mobile use & $\geq 2 \mathrm{~h} / \mathrm{d}$ & 154 & 4.9 \\
& $<30 \mathrm{~min} / \mathrm{d}$ & 1239 & 38.9 \\
\hline \multirow{2}{*}{ Physical activity } & $\geq 30 \mathrm{~min} / \mathrm{d}$ & 1943 & 61.1 \\
\hline
\end{tabular}


Table 4. Psychological Behaviors Among Iranian Adolescent Girls

\begin{tabular}{|c|c|c|c|}
\hline & & No. & $\%$ \\
\hline \multirow{2}{*}{ Worthlessness } & Low & 2687 & 84.9 \\
\hline & High & 478 & 15.1 \\
\hline \multirow{2}{*}{ Anger } & Low & 1808 & 57.2 \\
\hline & High & 1354 & 42.8 \\
\hline \multirow{2}{*}{ Worry } & Low & 1966 & 62 \\
\hline & High & 1206 & 38 \\
\hline \multirow{2}{*}{ Insomnia } & Low & 2411 & 76.3 \\
\hline & High & 747 & 23.7 \\
\hline \multirow{2}{*}{ Fatigue } & Low & 2876 & 90.9 \\
\hline & High & 288 & 9.1 \\
\hline \multirow{2}{*}{ Depression } & Low & 2541 & 80 \\
\hline & High & 637 & 20 \\
\hline \multirow{2}{*}{ Anxiety } & Low & 2930 & 92.6 \\
\hline & High & 234 & 7.4 \\
\hline \multirow{2}{*}{ Self-perceived health } & Good & 2510 & 78.9 \\
\hline & Poor & 670 & 21.1 \\
\hline \multirow{2}{*}{ Life satisfaction } & Satisfy & 2482 & 77.6 \\
\hline & Dissatisfy & 716 & 22.4 \\
\hline \multirow{3}{*}{ Weight perception } & Underestimate & 900 & 28.3 \\
\hline & Right weight & 1369 & 43.1 \\
\hline & Overestimate & 908 & 28.6 \\
\hline
\end{tabular}

documented from 2007 to 2016. This study reported that the prevalence rates of obesity and severe obesity were $20.6 \%$ and $7.7 \%$, respectively in $2016 .{ }^{16}$ In recent decades, changes in dietary patterns, including increased intake of soft drinks and sweets, as well as decreased intake of fruits and vegetables in many countries have led to a rapid rise in the prevalence of excess weight in childhood and adolescence. In spite of the changes from healthy diet to unhealthy dietary habits, some studies have not found any association between obesity and intake of fruits, vegetables, and soft drinks. There was a significant reverse association between consumption of sweets and body mass index in 31 out of the 34 countries that were examined (91\%). ${ }^{17}$ Increasing sweet consumption was correlated with decreasing likelihood of overweight. A possible explanation is that underreporting unhealthy food intake may be more common in overweight people in comparison with normal weight people. Another possibility is that the youth who consume sweets, eat less fatty foods including potato chips and pastries. Therefore, as fats have more caloric density compared with carbohydrates, their total caloric intake might have been decreased. ${ }^{17}$

The current study showed that $27.1 \%$ of Iranian adolescent girls slept for less than 8 hours a day. Short sleep duration and poor sleep quality are very common among adolescents. There is significant change in circadian rhythm in adolescents due to changes in their lifestyle, academic concerns, growing time of cellphone usage, participating in social programs and decreasing parental supervision. The prevalence of sleep problems during adolescence is about $25 \%$ to $50 \% .{ }^{18}$ The prevalence of insomnia among adolescents was $14.9 \%$ to $27 \%$ based on the accuracy of insomnia definition. ${ }^{19}$

Decreasing sleep hours is common in adolescents in both developed and developing countries. Sleep problems cause several mental health diseases. They lead to poorer school performance, problems in cognitive development, several physical problems, and chronic diseases throughout life. These facts indicate the importance of sleep hygiene during adolescence..$^{20,21}$

Table 5. Frequency of Food Items Intake And Lifestyle Habits According to the Socio-economic Status

\begin{tabular}{|c|c|c|c|c|c|}
\hline & & \multicolumn{3}{|c|}{ Socio-economic Status } & \multirow{2}{*}{$P$ Value } \\
\hline & & Low & Medium & High & \\
\hline \multirow{2}{*}{ Fruits } & Daily & $498,59.1 \%$ & $532,61.8 \%$ & $575,61.9 \%$ & \multirow{2}{*}{0.401} \\
\hline & Non-daily & $345,40.9 \%$ & $329,38.2 \%$ & $354,38.1 \%$ & \\
\hline \multirow{2}{*}{ Vegetables } & Daily & $319,32.1 \%$ & $336,33.8 \%$ & $361,34.8 \%$ & \multirow{2}{*}{0.443} \\
\hline & Non-daily & $674,67.9 \%$ & $659,66.2 \%$ & $677,65.2 \%$ & \\
\hline \multirow{2}{*}{ Dairy products } & Daily & $377,37.5 \%$ & $367,36.7 \%$ & $420,39.8 \%$ & \multirow{2}{*}{0.327} \\
\hline & Non-daily & $629,62.5 \%$ & $634,63.3 \%$ & $636,60.2 \%$ & \\
\hline \multirow{2}{*}{ Fast foods } & Daily & $118,11.7 \%$ & $106,10.6 \%$ & $101,9.6 \%$ & \multirow{2}{*}{0.291} \\
\hline & Non-daily & $888,88.3 \%$ & $896,89.4 \%$ & $952,90.4 \%$ & \\
\hline \multirow{2}{*}{ Sweets snacks } & Daily & $222,22.0 \%$ & $213,21.3 \%$ & $224,23.1 \%$ & \multirow{2}{*}{0.612} \\
\hline & Non-daily & $785,78.0 \%$ & $789,78.7 \%$ & $813,76.9 \%$ & \\
\hline \multirow{2}{*}{ Salty snacks } & Daily & $73,7.3 \%$ & $60,6.0 \%$ & $62,5.9 \%$ & \multirow{2}{*}{0.360} \\
\hline & Non-daily & $929,92.7 \%$ & $941,94.0 \%$ & $991,94.1 \%$ & \\
\hline \multirow{2}{*}{ Carbonated drinks } & Daily & $39,3.9 \%$ & $27,2.7 \%$ & $31,2.9 \%$ & \multirow{2}{*}{0.283} \\
\hline & Non-daily & $968,96.1 \%$ & $973,97.3 \%$ & $1025,97.1 \%$ & \\
\hline \multirow{2}{*}{ Physical activity } & $\operatorname{Low}(<30 \mathrm{~min} / \mathrm{d})$ & $624,64.1 \%$ & $612,61.3 \%$ & $601,57.6 \%$ & \multirow{2}{*}{$0.011^{*}$} \\
\hline & High ( $\geq 30 \mathrm{~min} / \mathrm{d})$ & $359,35.9 \%$ & $386,38.7 \%$ & $443,42.4 \%$ & \\
\hline \multirow{2}{*}{ Screen time } & $\operatorname{Low}(<2 \mathrm{~h} / \mathrm{d})$ & $826,84.1 \%$ & $814,82.9 \%$ & $839,81.1 \%$ & \multirow{2}{*}{0.211} \\
\hline & High $(\geq 2 \mathrm{~h} / \mathrm{d})$ & $156,15.9 \%$ & $168,17.1 \%$ & $195,18.9 \%$ & \\
\hline
\end{tabular}


The present study showed that $33.5 \%$ of Iranian adolescent girls had daily vegetable intake, and 60.9\% consumed fruits everyday. A Study in the USA showed that $28.5 \%$ of the middle school students did not consume fruit daily and $33.2 \%$ of students did not consume vegetables daily. ${ }^{22}$ Sufficient intake of fruits and vegetables is important because they are efficient sources of antioxidants and they also have an important role in prevention of non-communicable diseases. ${ }^{23}$

Our findings showed that $45.6 \%$ of Iranian adolescent girls consumed dairy products everyday. Dairy products are an important part of a healthy diet. They have a significant role in preventing obesity, hypertension, cardiovascular disease and improvement of bone and tooth health. Dairy products are sources of calcium, which is a vital micronutrient during adolescence. ${ }^{24}$ It is recommended that adolescents should consume at least 3 servings of dairy products a day. A large study on American adolescent girls showed that more than $90 \%$ of them did not consume the proper amount of dairy per day. ${ }^{25}$ Furthermore, recent studies showed that the prevalence of dairy consumption has decreased in recent years, whereas consumption of soft drinks and juices has increased among adolescent girls. ${ }^{26}$

According to our findings, fast foods were consumed $10.6 \%$ daily and $16.2 \%$ weekly by Iranian adolescent girls. Obesity is one of the major concerns in the adolescence period. Consumption of fast foods and snacks is increasing in modern life and it is an undeniable reason for this problem. ${ }^{27}$ Adolescents prefer to spend their time with people of the same age and they sometimes unintentionally consume fast foods because they want to spend time with their friends in fast food restaurants. Studies in Western countries showed that the prevalence of weekly fast food consumption was more than $70 \%{ }^{28,29}$ Lower prevalence of fast food consumption among Iranian adolescent girls compared to Western countries is probably due to the socio-cultural differences, especially in rural areas of different countries with less spending of time with friends in fast food restaurants.

We found that $84.2 \%$ of Iranian adolescent girls consumed breakfast regularly; it is higher than breakfast consumption in Western countries which is reported to be about $60 \%$ to $70 \% .^{30,31}$ Breakfast is the most important meal of the day, and has a significant effect on physical and cognitive development during the adolescence period. A growing body of evidence has documented the effects of breakfast consumption on reducing the risk of obesity and overweight. ${ }^{32}$ A study in rural and urban South African adolescents reported that $60 \%$ of participants had regular breakfast intake. ${ }^{33}$ Likewise, a study in Turkey reported that $77.9 \%$ of students had breakfast every day. ${ }^{34}$

In this study, we found that $61.1 \%$ of Iranian adolescent girls had physical activity for more than 30 minutes per day. In 2010, the WHO reported that only $16 \%$ of female adolescents are physically active worldwide.
Physical activity is one of the other significant parts of a healthy lifestyle. In recent years, the amount of physical activity has diminished in both developed and developing countries. Adolescents, especially female adolescents due to academic competitions and also participating in other social programs, have less time for healthy and sufficient physical activity. ${ }^{35}$ Increasing the time spent on watching television, use of personal computers, and a reduction in physical activity in schools and communities have resulted in considerable change in the pattern of physical activity. American guidelines suggest 30-60 minutes of physical activity for young people on most or all days of the week. The Great Britain guidelines suggest at least 60 minutes of physical activity on at least 5 days a week. Canadian guidelines suggest gradually increasing daily physical activity to 90 minutes for adolescents. ${ }^{36,37}$ Physical activity levels among adolescents have been reported widely in different countries but most of them do not meet the sufficient levels. ${ }^{38}$ In a large study on young German adults aged 14-24 years, Strohle et $\mathrm{al}^{39}$ showed that only $41 \%$ of female adolescents had physical activity regularly. They also reported that adolescents with higher physical activity had a lower risk for some mental illnesses like depression. Interventions in physical activity should be considered as an essential component for reducing the global obesity epidemic. Sedentary behaviors in youth can be reduced by increasing opportunities for more physical activity in schools and community.

The growing prevalence of obesity and physical inactivity in adolescents is directly related to the increasing hours of watching television and playing video games. Watching television not only causes a significant decrease in physical activity but can also increase the consumption of snacks and high-calorie foods. According to our findings, 55.2\% of Iranian adolescent girls spent more than 2 hours per day watching television. A study on American high school students reported that $42.8 \%$ of girls spent more than 2 hours a day watching television. ${ }^{40}$ This difference may be due to religious differences and the lack of suitable promenade for Iranian girls, so they prefer to stay home.

Increasing peak bone mass during the adolescence period prevents bone diseases in adulthood and senescence, especially osteoporosis, and causes a significant decrease in osteoporotic fractures. Considering the higher prevalence of osteoporosis in women, receiving sufficient vitamin $\mathrm{D}$ is an important issue in adolescent girls. Radiation of ultraviolet (UV) B wave band starts the primary phase of vitamin D3 synthesis in the human body and is the main source of vitamin D. ${ }^{41}$ We found that $16.3 \%$ of Iranian adolescent girls have less than 5 minutes of sun exposure per day and $60.2 \%$ receive sunlight for less than 30 minutes per day. In a study on adolescents in the southern part of Spain, Fernandez et $\mathrm{al}^{42}$ reported that only $11.1 \%$ of female adolescents received sunlight for less than 1 hour. Girls spent less time outdoors on weekends and received 
less sunlight than boys. The socio-cultural differences between Iran and Western countries such as Spain can be the reason for the difference in sun exposure.

According to studies, the greatest sun exposure occurs before the age of 20 among 13-19-year-old adolescents with longer exposure times on weekends and during summer vacation. Sunlight exposure is important for vitamin $\mathrm{D}$ synthesis. However, a high prevalence of vitamin $\mathrm{D}$ deficiency has been reported in sunny areas of Middle Eastern countries. Dietary consumption of vitamin D is essential when sun exposure is inadequate. However, dietary sources for providing the daily recommended requirement of vitamin $\mathrm{D}$ are low. One of the main factors that affect the percentage of the ground level of ultraviolet $\mathrm{B}$ (UVB) is air pollution. The amount of solar UVB that reaches the earth surface is inversely correlated with the amount of air pollution. Thus, air pollution should be considered as a risk factor for hypovitaminosis D. ${ }^{43,44}$

In the present study, there were no differences in intake of food items among adolescent girls according to their SES. Our findings are not consistent with a study on 162 schools (25309 students) in Greece which showed that children and adolescents in medium or high SES families had better diet quality with more consumption of fruits, vegetables, and dairy. ${ }^{45}$

The positive association between SES and being more physically active in the present study is in agreement with findings from some previous studies. ${ }^{46}$ Habitual physical activity level was lower among low SES Brazilian children. ${ }^{47}$ One study on 6-8-year-old girls found that supervised physical activity and organized sports increased with higher family income and education levels. Likewise, higher SES was associated with lower television time in German children and adolescents. ${ }^{48}$ However, in the current study, there was no difference in screen time according to the SES of participants.

Regional and national organizations and international agencies must cooperate for prevention of unhealthy lifestyle in adolescents. Physical activity and healthy diets should be encouraged in school-age youth. A new approach and modern tools are needed to promote changes in lifestyle of adolescents more effectively. Regarding proper weight control, healthcare staff should recommend healthy food items without any compulsion because adolescence is a unique period in life. Computer-tailored education is an efficient way for changing the lifestyle and increasing motivation for adopting a healthy diet and physical activity. According to the findings, computertailored education is more effective than general education in adolescents. ${ }^{49}$

In the present study, we assessed lifestyle factors and eating habits in a large sample of Iranian adolescent girls with different SES living in rural and urban areas. The large study population and their representativeness provide a realistic view on lifestyle habits of Iranian adolescent girls.
However, our study has some limitations. First, data of lifestyle variables and dietary habits were extracted from self-reported questionnaires, which probably reduces their accuracy. Second, we used 16 dietary groups for assessment of dietary habits without any information regarding the number of servings per day.

In conclusion, this large nationwide study showed that the consumption of vegetables, fruits, and dairy products was lower than desirable levels among Iranian adolescent girls. Skipping breakfast, sleep problems, as well as low physical activity, especially in those with high SES, were among major unhealthy lifestyle habits. As adolescence is an important period of life for girls, affecting their current wellbeing as well as their future health and the health of the next generation, health promotion and improvement of lifestyle habits of adolescent girls should be underscored in health programs at individual and public levels.

\section{Authors' Contribution}

$\mathrm{MHB}$ and RK equally contributed to the conception and design of the research; RK, MEM and RH contributed to the design of the research; RR contributed to the acquisition and analysis of the data; RSM, MHB, ShF and SShD contributed to the interpretation of the data; and RSM and MHB drafted the manuscript. All authors critically revised the manuscript, agree to be fully accountable for ensuring the integrity and accuracy of the work, and read and approved the final manuscript.

\section{Conflict of Interest Disclosures}

None.

\section{Ethical Statement}

The study protocol was approved by ethical committees and other relevant national regulatory organizations. The present study was approved by the Research and Ethics Council of Isfahan University of Medical Sciences. We explained the objectives and protocols of the study and after that, written informed consent was obtained from the students and their parents.

\section{References}

1. Patton GC, Sawyer SM, Santelli JS, Ross DA, Afifi R, Allen NB, et al. Our future: a Lancet commission on adolescent health and wellbeing. Lancet. 2016;387(10036):2423-78. doi: 10.1016/S0140-6736(16)00579-1..

2. Viner RM, Ozer EM, Denny S, Marmot M, Resnick M, Fatusi A, et al. Adolescence and the social determinants of health. Lancet. 2012;379(9826):1641-52. doi: 10.1016/S01406736(12)60149-4.

3. Mokdad AH, Forouzanfar MH, Daoud F, Mokdad AA, El Bcheraoui C, Moradi-Lakeh M, et al. Global burden of diseases, injuries, and risk factors for young people's health during 1990-2013: a systematic analysis for the Global Burden of Disease Study 2013. Lancet. 2016;387(10036):2383-401. doi: 10.1016/S0140-6736(16)00648-6

4. Blum LS, Mellisa A, Kurnia Sari E, Novitasari Yusadiredja I, van Liere M, Shulman S, et al. In-depth Assessment of Snacking Behavior in Unmarried Adolescent Girls Living in Urban Centers of Java, Indonesia. Matern Child Nutr. 2019;15(4):e12833. doi: 10.1111/mcn.12833.

5. Das JK, Salam RA, Thornburg KL, Prentice AM, Campisi S, Lassi ZS, et al. Nutrition in adolescents: physiology, metabolism, and nutritional needs. Ann N Y Acad Sci. 2017;1393(1):2133. doi: 10.1111/nyas.13330.

6. Brand S, Gerber M, Beck J, Hatzinger M, Puhse U, HolsboerTrachsler E. High exercise levels are related to favorable 
sleep patterns and psychological functioning in adolescents: a comparison of athletes and controls. J Adolesc Health. 2010;46(2):133-41doi: $\quad$ 10.1016/j.jadohealth.2009.06.018.

7. Richardson C, Oar E, Fardouly J, Magson N, Johnco C, Forbes $M$, et al. The Moderating Role of Sleep in the Relationship Between Social Isolation and Internalising Problems in Early Adolescence. Child Psychiatry Hum Dev. 2019;50(6):10111020. doi: 10.1007/s10578-019-00901-9.

8. Akseer N, Al-Gashm S, Mehta S, Mokdad A, Bhutta ZA. Global and regional trends in the nutritional status of young people: a critical and neglected age group. Ann N Y Acad Sci. 2017;1393(1):3-20. doi: 10.1111/nyas.13336.

9. Webb AR, Kift R, Durkin MT, O'Brien SJ, Vail A, Berry JL, et al. The role of sunlight exposure in determining the vitamin $\mathrm{D}$ status of the U.K. white adult population. $\mathrm{Br} J$ Dermatol. 2010;163(5):1050-5. doi: 10.1111/j.1365-2133.2010.09975.x.

10. Motlagh ME, Ziaodini H, Qorbani M, Taheri M, Aminaei T, Goodarzi A, et al. Methodology and early findings of the fifth survey of childhood and adolescence surveillance and prevention of adult noncommunicable disease: The CASPIAN-V Study. Int J Prev Med. 2017;8:4. doi: 10.4103/20087802.198915

11. Kelishadi R, Motlagh M, Roomizadeh P, Abtahi S, Qorbani M, Taslimi M, et al. First report on path analysis for cardiometabolic components in a nationally representative sample of pediatric population in the Middle East and North Africa (MENA): the CASPIAN-III Study. Ann Nutr Metab. 2013;62(3):257-65. doi: 10.1159/000346489.

12. De Stefani E, Boffetta P, Ronco AL, Deneo-Pellegrini H, Acosta $\mathrm{G}$, Gutierrez LP, et al. Nutrient patterns and risk of lung cancer: a factor analysis in Uruguayan men. Lung Cancer 2008;61(3):283-91. doi: 10.1016/j.lungcan.2008.01.004.

13. Janssen I, Leblanc A. Systematic review of the health benefits of physical activity and fitness in school-aged children and youth. Int J Behav Nutr Phys Act. 2010;7:40. doi: 10.1186 / 14795868-7-40

14. Mazidi M, Banach M, Kengne AP. Prevalence of childhood and adolescent overweight and obesity in Asian countries: a systematic review and meta-analysis. Arch Med Sci. 2018;14(6):1185-1203. doi: 10.5114/aoms.2018.79001.

15. Ogden CL, Carroll MD, Kit BK, Flegal KM. Prevalence of obesity and trends in body mass index among US children and adolescents, 1999-2010. JAMA. 2012;307(5):483-90. doi: 10.1001/jama.2012.40.

16. Hales CM, Fryar CD, Carroll MD, Freedman DS, Ogden CL. Trends in Obesity and Severe Obesity Prevalence in US Youth and Adults by Sex and Age, 2007-2008 to 2015-2016. JAMA. 2018;319(16):1723-1725. doi: 10.1001/jama.2018.3060.

17. Moreno LA, Gonzalez-Gross M, Kersting M, Molnar D, de Henauw S, Beghin L, et al. Assessing, understanding and modifying nutritional status, eating habits and physical activity in European adolescents: the HELENA (Healthy Lifestyle in Europe by Nutrition in Adolescence) Study. Public Health Nutr. 2008;11(3):288-99. doi: 10.1017/S1368980007000535.

18. Carra MC. Sleep bruxism and sleep disorders in adolescents. J Dentofacial Anom Orthod. 2018;21(1):108-118.

19. Combs D, Goodwin JL, Quan SF, Morgan WJ, Shetty S, Parthasarathy S. Insomnia, health-related quality of life and health outcomes in children: a seven year longitudinal cohort. Sci Rep. 2016;6:27921. doi: 10.1038/srep27921.

20. Murugesan G, Karthigeyan L, Selvagandhi PK, Gopichandran V. Sleep patterns, hygiene and daytime sleepiness among adolescent school-goers in three districts of Tamil Nadu: a descriptive study. Natl Med J India. 2018;31(4):196-200. doi: 10.4103/0970-258X.258216.

21. Chen T, Wu Z, Shen Z, Zhang J, Shen X, Li S. Sleep duration in Chinese adolescents: biological, environmental, and behavioral predictors. Sleep Med. 2014;15(11):1345-53. doi: 10.1016/j. sleep.2014.05.018.

22. Centers for Disease Control and Prevention (CDC). Fruit and vegetable consumption among high school students-United States, 2010. MMWR Morb Mortal Wkly Rep. 2011;60(46):1583-6

23. Pem D, Jeewon R. Fruit and Vegetable Intake: Benefits and Progress of Nutrition Education Interventions- Narrative Review Article. Iran J Public Health. 2015;44(10):1309-21.

24. Racey M, Bransfield J, Capello K, Field D, Kulak V, Machmueller $\mathrm{D}$, et al. Barriers and facilitators to intake of dairy products in adolescent males and females with different levels of habitual intake. Glob Pediatr Health. 2017;4:2333794X17694227. doi: $10.1177 / 2333794 \times 17694227$.

25. Moore LL, Singer MR, Qureshi MM, Bradlee ML, Daniels SR. Food group intake and micronutrient adequacy in adolescent girls. Nutrients. 2012;4(11):1692-708. doi: 10.3390/ nu4111692.

26. Vartanian LR, Schwartz MB, Brownell KD. Effects of soft drink consumption on nutrition and health: a systematic review and meta-analysis. Am J Public Health. 2007;97(4):667-75. doi: 10.2105/AJPH.2005.083782.

27. Chapman G, Maclean H. "Junk food" and "healthy food": meanings of food in adolescent women's culture. J Nutr Educ. 1993;25(3):108-13.

28. French SA, Story M, Neumark-Sztainer D, Fulkerson JA, Hannan P. Fast food restaurant use among adolescents: associations with nutrient intake, food choices and behavioral and psychosocial variables. Int J Obes Relat Metab Disord. 2001;25(12):1823-33. doi: 10.1038/sj.ijo.0801820.

29. Morse KL, Driskell JA. Observed sex differences in fast-food consumption and nutrition self-assessments and beliefs of college students. Nutr Res. 2009;29(3):173-9. doi: 10.1016/j. nutres.2009.02.004.

30. Hallstrom L, Vereecken CA, Labayen I, Ruiz JR, Le Donne C, Garcia MC, et al. Breakfast habits among European adolescents and their association with sociodemographic factors: the HELENA (Healthy Lifestyle in Europe by Nutrition in Adolescence) study. Public Health Nutr. 2012;15(10):1879-89. doi: $\quad 10.1017 / S 1368980012000341$.

31. Rampersaud GC, Pereira MA, Girard BL, Adams J, Metzl JD. Breakfast habits, nutritional status, body weight, and academic performance in children and adolescents. J Am Diet Assoc. 2005;105(5):743-60; quiz 761-2. doi: 10.1016/j. jada.2005.02.007

32. Szajewska H, Ruszczynski M. Systematic review demonstrating that breakfast consumption influences body weight outcomes in children and adolescents in Europe. Crit Rev Food Sci Nutr. 2010;50(2):113-9. doi: 10.1080/10408390903467514.

33. Sedibe MH, Pisa PT, Feeley AB, Pedro TM, Kahn K, Norris SA. Dietary habits and eating practices and their association with overweight and obesity in rural and urban Black South African adolescents. Nutrients. 2018;10(2):145. doi: 10.3390/ nu10020145

34. Erenoglu N, Ayranci U, Son O. Eating habits reported by secondary school students in a city of west Turkey. Eat Behav. 2006;7(4):348-54. doi: 10.1016/j.eatbeh.2005.11.009.

35. Kohl HW, 3rd, Craig CL, Lambert EV, Inoue S, Alkandari JR, Leetongin G, et al. The pandemic of physical inactivity: global action for public health. Lancet. 2012;380(9838):294-305. doi: 10.1016/S0140-6736(12)60898-8.

36. Piercy KL, Troiano RP, Ballard RM, Carlson SA, Fulton JE, Galuska DA, et al. The Physical Activity Guidelines for Americans Physical Activity Guidelines for Americans Physical Activity Guidelines for Americans. JAMA. 2018;320(19):20202028. doi: 10.1001/jama.2018.14854.

37. Janssen I, Katzmarzyk PT, Boyce WF, Vereecken C, Mulvihill C, Roberts C, et al. Comparison of overweight and obesity prevalence in school-aged youth from 34 countries and their 
relationships with physical activity and dietary patterns. Obes Rev. 2005;6(2):123-32. doi: 10.1111/j.1467789x.2005.00176.x.

38. Carson V, Spence JC. Seasonal variation in physical activity among children and adolescents: a review. Pediatr Exerc Sci. 2010;22(1):81-92

39. Ströhle A, Höfler M, Pfister H, Müller Ag, Hoyer J, Wittchen $\mathrm{H}-\mathrm{U}$, et al. Physical activity and prevalence and incidence of mental disorders in adolescents and young adults. Psychol Med. 2007;37(11):1657-66. doi: 10.1017/S003329170700089X.

40. Lowry R, Wechsler H, Galuska DA, Fulton JE, Kann L. Television viewing and its associations with overweight, sedentary lifestyle, and insufficient consumption of fruits and vegetables among US high school students: differences by race, ethnicity, and gender. J Sch Health. 2002;72(10):413-21. doi: 10.1111/j.1746-1561.2002.tb03551.x.

41. Holick MF. Biological Effects of Sunlight, Ultraviolet Radiation, Visible Light, Infrared Radiation and Vitamin D for Health. Anticancer Res. 2016;36(3):1345-56.

42. Fernandez-Morano T, De Troya-Martin M, Rivas-Ruiz F, Blazquez-Sanchez N, Del Boz-Gonzalez J, Fernandez-Penas $P$, et al. Behaviour, attitudes and awareness concerning sun exposure in adolescents on the Costa del Sol. Eur J Dermatol. 2014;24(1):85-93. doi: 10.1684/ejd.2014.2266.

43. Kelishadi R, Moeini R, Poursafa P, Farajian S, Yousefy H, Okhovat-Souraki AA. Independent association between air pollutants and vitamin $\mathrm{D}$ deficiency in young children in Isfahan, Iran. Paediatr Int Child Health. 2014;34(1):50-5. doi: 10.1179/2046905513Y.0000000080.
44. Kelishadi R, Sharifi-Ghazvini F, Poursafa P, Mehrabian F, Farajian S, Yousefy H, et al. Determinants of hypovitaminosis $\mathrm{d}$ in pregnant women and their newborns in a sunny region. Int J Endocrinol. 2013;2013:460970. doi: 10.1155/2013/460970.

45. Yannakoulia M, Lykou A, Kastorini CM, Saranti Papasaranti E, Petralias A, Veloudaki A, et al. Socio-economic and lifestyle parameters associated with diet quality of children and adolescents using classification and regression tree analysis: the DIATROFI study. Public Health Nutr. 2016;19(2):339-47. doi: 10.1017/S136898001500110X.

46. Lampinen EK, Eloranta AM, Haapala EA, Lindi V, Vaisto J, Lintu N, et al. Physical activity, sedentary behaviour, and socioeconomic status among Finnish girls and boys aged 6-8 years. Eur J Sport Sci. 2017;17(4):462-472. doi: 10.1080/17461391.2017.1294619.

47. dos Santos Amorim P, de Faria R, Byrne N, Hills A. Physical activity and nutritional status of Brazilian children of low socioeconomic status: undernutrition and overweight. BMC Health Serv Res. 2016;16(suppl 3):200. doi: 10.1186/s12913016-1423-5.

48. Poulain T, Vogel M, Sobek C, Hilbert A, Korner A, Kiess W. Associations between socio-economic status and child health: findings of a large German cohort study. Int J Environ Res Public Health. 2019;16(5):677. doi: 10.3390/ijerph16050677.

49. Broekhuizen K, Kroeze W, van Poppel MN, Oenema A, Brug J. A systematic review of randomized controlled trials on the effectiveness of computer-tailored physical activity and dietary behavior promotion programs: an update. Ann Behav Med. 2012;44(2):259-86. doi: 10.1007/s12160-012-9384-3. 